\title{
A new approach for one-dimensional sine-Gordon equation
}

\author{
Ali Akgül ${ }^{* *} \mathbb{D}$, Mustafa Inc ${ }^{2}$, Adem Kilicman ${ }^{3}$ and Dumitru Baleanu ${ }^{4,5}$
}

"Correspondence: aliakgul00727@gmail.com

${ }^{1}$ Department of Mathematics, Art and Science Faculty, Siirt University, Siirt, 56100, Turkey

Full list of author information is available at the end of the article

\begin{abstract}
In this work, we use a reproducing kernel method for investigating the sine-Gordon equation with initial and boundary conditions. Numerical experiments are studied to show the efficiency of the technique. The acquired results are compared with the exact solutions and results obtained by different methods. These results indicate that the reproducing kernel method is very effective.
\end{abstract}

Keywords: reproducing kernel method; sine-Gordon equation; bounded linear operator; homogenizing

\section{Introduction}

The nonlinear one-dimensional sine-Gordon (SG) equation came into sight in the differential geometry and attracted a lot of attention because of the collisional behaviors of solitons that arise from this equation. Numerical solutions of the SG equation have been widely investigated in recent years [1-5]. Compact finite difference and diagonally implicit Runge-Kutta-Nyström (DIRKN) methods were used [3]. The authors of [6] introduced a numerical method for solving the SG equation by using collocation and radial basis functions. The boundary integral equation technique is presented in [7]. Bratsos [8,9] has researhed a numerical technique for solving the one-dimensional SG equation and a thirdorder numerical technique for the two-dimensional SG equation. A numerical technique using radial basis functions for the solution of the two-dimensional SG equation has been shown in [10]. Some authors advised spectral techniques and Fourier pseudospectral technique for solving nonlinear wave equation taking a discrete Fourier series and Chebyshev orthogonal polynomials [11-13]. Ma and Wu [14] used a meshless technique by using a multiquadric $(\mathrm{MQ})$ quasi-interpolation. In this paper, we investigate the one-dimensional nonlinear sine-Gordon equation

$$
\frac{\partial^{2} u}{\partial \tau^{2}}(\eta, \tau)=\frac{\partial^{2} u}{\partial \eta^{2}}(\eta, \tau)-\sin (u(\eta, \tau)), \quad 0 \leq \eta \leq 1, \tau \geq 0
$$

with initial conditions

$$
\begin{aligned}
& u(\eta, 0)=f(\eta), \quad 0 \leq \eta \leq 1, \\
& \frac{\partial u}{\partial \tau}(\eta, 0)=g(\eta), \quad 0 \leq \eta \leq 1,
\end{aligned}
$$

(C) 2016 Akgül et al. This article is distributed under the terms of the Creative Commons Attribution 4.0 International License (http://creativecommons.org/licenses/by/4.0/), which permits unrestricted use, distribution, and reproduction in any medium, provided you give appropriate credit to the original author(s) and the source, provide a link to the Creative Commons license, and indicate if changes were made. 
and boundary conditions

$$
u(0, \tau)=h_{1}(\tau), \quad u(1, \tau)=h_{2}(\tau), \quad \tau \geq 0,
$$

by using the reproducing kernel method (RKM). We can get numerical results in very short time. By this method nonlinear problems can be solved easily like linear problems. Reproducing kernel functions are very important for numerical results. We can change the inner product in the spaces and obtain different reproducing kernel functions for better results. These are advantages of this method. Homogenizing the initial and boundary conditions is very significant for this method. We give a general transformation to homogenize the initial and boundary conditions in Section 3.

The theory of reproducing kernels [15] was used for the first time at the beginning of the 20th century by Zaremba. Reproducing kernel theory has important implementations in numerical analysis, differential equations, and probability and statistics [16-25]. The efficiency of the method was used by many authors to research several scientific implementations. The reproducing kernel functions can be represented by piecewise polynomials, and the higher the order of derivatives, the simpler the reproducing kernel function statements. Such statements of reproducing kernel functions are the simplest from the computational viewpoint, and the speed and accuracy can be significantly improved in scientific and engineering implementations. The productivity of such reproducing kernel functions is indicated to be very exhorting by experimental results [26].

This work is arranged as follows. Section 2 introduces several useful reproducing kernel functions. A representation of solution in $W_{2}^{(3,3)}(\Omega)$ is given in Section 3. Section 4 presents the essential results: exact and approximate solutions of (1)-(3); enhancement of the method to some problems in the reproducing kernel space; and convergence of the approximate solution. Some numerical examples are discussed in Section 5. There are some conclusions in the final section.

\section{Reproducing kernel functions}

We obtain some useful reproducing kernel functions in this section.

Definition 1 [16] Let $E$ be a nonempty set. A function $K: E \times E \rightarrow C$ is called a reproducing kernel function of the Hilbert space $H$ if

(a) $\forall \tau \in E, K(\cdot, \tau) \in H$,

(b) $\forall \tau \in E, \forall \varphi \in H,\langle\varphi(\cdot), K(\cdot, \tau)\rangle=\varphi(\tau)$.

Definition 2 [16] A Hilbert space $H$ defined on a nonempty set $E$ is called a reproducing kernel Hilbert space if there exists a reproducing kernel function $K(\eta, \tau)$.

Definition 3 [16] We define the $W_{2}^{3}[0,1]$ by

$$
W_{2}^{3}[0,1]=\left\{\begin{array}{c}
u \mid u, u^{\prime}, u^{\prime \prime} \text { are absolutely continuous real-valued functions in }[0,1], \\
u^{(3)} \in L^{2}[0,1], \eta \in[0,1], u(0)=0, u(1)=0
\end{array}\right\} .
$$

The inner product and the norm in $W_{2}^{3}[0,1]$ are defined respectively by

$$
\langle u, v\rangle_{W_{2}^{3}}=u(0) v(0)+u^{\prime}(0) v^{\prime}(0)+u^{\prime}(1) v^{\prime}(1)+\int_{0}^{1} u^{(3)}(\eta) v^{(3)}(\eta) d \eta, \quad u, v \in W_{2}^{3}[0,1]
$$


and

$$
\|u\|_{W_{2}^{3}}=\sqrt{\langle u, u\rangle_{W_{2}^{3}}}, \quad u \in W_{2}^{3}[0,1] .
$$

Definition 4 [16] We define the space $F_{2}^{3}[0, T]$ by

$$
F_{2}^{3}[0, T]=\left\{\begin{array}{c}
u \mid u, u^{\prime}, u^{\prime \prime} \text { are absolutely continuous in }[0, T], \\
u^{(3)} \in L^{2}[0, T], \tau \in[0, T], u(0)=0, u^{\prime}(0)=0
\end{array}\right\}
$$

with the inner product and norm

$$
\langle u, v\rangle_{F_{2}^{3}}=\sum_{i=0}^{2} u^{(i)}(0) v^{(i)}(0)+\int_{0}^{1} u^{(3)}(T) v^{(3)}(\tau) d \tau, \quad u, v \in F_{2}^{3}[0, T]
$$

and

$$
\|u\|_{F_{2}^{3}}=\sqrt{\langle u, u\rangle_{F_{2}^{3}}}, \quad u \in F_{2}^{3}[0, T] .
$$

The space $F_{2}^{3}[0, T]$ is a reproducing kernel space, and its reproducing kernel function $r_{s}$ is given by

$$
r_{s}(\tau)= \begin{cases}\frac{1}{4} s^{2} \tau^{2}+\frac{1}{12} s^{2} \tau^{3}-\frac{1}{24} s \tau^{4}+\frac{1}{120} \tau^{5}, & \tau \leq s, \\ \frac{1}{4} s^{2} \tau^{2}+\frac{1}{12} s^{3} \tau^{2}-\frac{1}{24} \tau s^{4}+\frac{1}{120} s^{5}, & \tau>s .\end{cases}
$$

Definition 5 [16] We define the space $H_{2}^{1}[0, T]$ by

$$
H_{2}^{1}[0, T]=\left\{\begin{array}{c}
u \mid u \text { is absolutely continuous in }[0,1], \\
u^{\prime} \in L^{2}[0, T], \tau \in[0, T]
\end{array}\right\}
$$

the inner product and norm

$$
\langle u, v\rangle_{H_{2}^{1}}=u(0) v(0)+\int_{0}^{\tau} u^{\prime}(T) v^{\prime}(\tau) d \tau, \quad u, v \in H_{2}^{1}[0, T]
$$

and

$$
\|u\|_{H_{2}^{1}}=\sqrt{\langle u, u\rangle_{H_{2}^{1}}}, \quad u \in H_{2}^{1}[0, T]
$$

Its reproducing kernel function $q_{s}$ is

$$
q_{s}(\tau)= \begin{cases}1+\tau, & \tau \leq s \\ 1+s, & \tau>s\end{cases}
$$

Definition 6 [16] We define the space $G_{2}^{1}[0,1]$ by

$$
G_{2}^{1}[0,1]=\left\{\begin{array}{c}
u \mid u \text { is absolutely continuous in }[0,1], \\
u^{\prime} \in L^{2}[0,1], \eta \in[0,1]
\end{array}\right\}
$$


with the inner product and norm

$$
\langle u, v\rangle_{G_{2}^{1}}=u(0) v(0)+\int_{0}^{1} u^{\prime}(\eta) v^{\prime}(\eta) d \eta, \quad u, v \in G_{2}^{1}[0,1]
$$

and

$$
\|u\|_{G_{2}^{1}}=\sqrt{\langle u, u\rangle_{G_{2}^{1}}}, \quad u \in G_{2}^{1}[0,1] .
$$

The space $G_{2}^{1}[0,1]$ is a reproducing kernel space, and its reproducing kernel function $Q_{y}$ is given by

$$
Q_{y}(\eta)= \begin{cases}1+\eta, & \eta \leq y, \\ 1+y, & \eta>y .\end{cases}
$$

Theorem 2.1 The reproducing kernel function $R_{y}$ of $W_{2}^{3}[0,1]$ is

$$
R_{y}(\eta)= \begin{cases}\sum_{i=1}^{6} c_{i}(y) \eta^{i-1}, & \eta \leq y, \\ \sum_{i=1}^{6} d_{i}(y) \eta^{i-1}, & \eta>y\end{cases}
$$

where

$$
\begin{aligned}
& c_{1}(y)=0, \quad c_{4}(y)=0, \quad d_{1}(y)=\frac{1}{120} y^{5}, \quad d_{4}(y)=-\frac{1}{12} y^{2}, \\
& c_{2}(y)=-\frac{1}{122} y^{5}+\frac{5}{244} y^{4}-\frac{127}{244} y^{2}+\frac{31}{61} y, \\
& c_{3}(y)=-\frac{1}{2,928} y^{5}+\frac{127}{5,856} y^{4}-\frac{1}{12} y^{3}+\frac{1,137}{1,952} y^{2}-\frac{127}{244} y, \\
& c_{5}(y)=\frac{1}{2,938} y^{5}-\frac{5}{5,856} y^{4}+\frac{127}{5,856} y^{2}-\frac{31}{1,464} y, \\
& c_{6}(y)=-\frac{1}{7,320} y^{5}+\frac{1}{2,928} y^{4}-\frac{1}{2,928} y^{2}-\frac{1}{122} y+\frac{1}{120} \\
& d_{2}(y)=-\frac{1}{122} y^{5}-\frac{31}{1,464} y^{4}-\frac{127}{244} y^{2}+\frac{31}{61} y, \\
& d_{3}(y)=-\frac{1}{2,928} y^{5}+\frac{127}{5,856} y^{4}+\frac{1,137}{1,952} y^{2}-\frac{127}{244} y, \\
& d_{5}(y)=\frac{1}{2,928} y^{5}-\frac{5}{5,856} y^{4}+\frac{127}{5,856} y^{2}+\frac{5}{244} y, \\
& d_{6}(y)=-\frac{1}{7,320} y^{5}+\frac{1}{2,928} y^{4}-\frac{1}{2,928} y^{2}-\frac{1}{122} y .
\end{aligned}
$$

Proof Let $u \in W_{2}^{3}[0,1]$ and $0 \leq y \leq 1$. Define $R_{y}$ by (4). Note that

$$
\begin{aligned}
& R_{y}^{\prime}(\eta)= \begin{cases}\sum_{i=1}^{5} i c_{i+1}(y) \eta^{i-1}, & \eta<y, \\
\sum_{i=1}^{5} i d_{i+1}(y) \eta^{i-1}, & \eta>y,\end{cases} \\
& R_{y}^{\prime \prime}(\eta)= \begin{cases}\sum_{i=1}^{4} i(i+1) c_{i+2}(y) \eta^{i-1}, & \eta<y, \\
\sum_{i=1}^{4} i(i+1) d_{i+2}(y) \eta^{i-1}, & \eta>y,\end{cases}
\end{aligned}
$$




$$
\begin{aligned}
& R_{y}^{(3)}(\eta)= \begin{cases}\sum_{i=1}^{3} i(i+1)(i+2) c_{i+3}(y) \eta^{i-1}, & \eta<y, \\
\sum_{i=1}^{3} i(i+1)(i+2) d_{i+3}(y) \eta^{i-1}, & \eta>y,\end{cases} \\
& R_{y}^{(4)}(\eta)= \begin{cases}\sum_{i=1}^{2} i(i+1)(i+2)(i+3) c_{i+4}(y) \eta^{i-1}, & \eta<y, \\
\sum_{i=1}^{2} i(i+1)(i+2)(i+3) d_{i+4}(y) \eta^{i-1}, & \eta>y,\end{cases}
\end{aligned}
$$

and

$$
R_{y}^{(5)}(\eta)= \begin{cases}120 c_{6}(y), & \eta<y \\ 120 d_{6}(y), & \eta>y\end{cases}
$$

By Definition 5 and integration by parts we have

$$
\begin{aligned}
\left\langle u(\eta), R_{y}(\eta)\right\rangle_{W_{2}^{3}}= & u(0) R_{y}(0)+u^{\prime}(0) R_{y}^{\prime}(0)+u^{\prime}(1) R_{y}^{\prime}(1)+u^{\prime \prime}(1) R_{y}^{(3)}(1) \\
& -u^{\prime \prime}(0) R_{y}^{(3)}(0)-u^{\prime}(1) R_{y}^{(4)}(1)+u^{\prime}(0) R_{y}^{(4)}(0)+\int_{0}^{1} u^{\prime}(\eta) R_{y}^{(5)}(\eta) d \eta \\
= & u^{\prime}(0)\left(R_{y}^{\prime}(0)+R_{y}^{(4)}(0)\right)+u^{\prime}(1)\left(R_{y}^{\prime}(1)-R_{y}^{(4)}(1)\right) \\
& +u^{\prime \prime}(1) R_{y}^{(3)}(1)-u^{\prime \prime}(0) R_{y}^{(3)}(0) \\
& +\int_{0}^{y} R_{y}^{(5)}(\eta) u^{\prime}(\eta) d \eta+\int_{y}^{1} R_{y}^{(5)}(\eta) u^{\prime}(\eta) d \eta \\
= & u^{\prime}(0)\left(c_{2}(y)+24 c_{5}(y)\right)-u^{\prime \prime}(0)\left(6 c_{4}(y)\right) \\
& +u^{\prime}(1)\left(d_{2}(y)+2 d_{3}(y)+3 d_{4}(y)-20 d_{5}(y)-115 d_{6}(y)\right) \\
& +u^{\prime \prime}(1)\left(6 d_{4}(y)+24 d_{5}(y)+60 d_{6}(y)\right) \\
& +\int_{0}^{y} 120 c_{6}(y) u(\eta) d \eta+\int_{y}^{1} 120 d_{6}(y) u(\eta) d \eta \\
= & 120 u(y)\left(\frac{1}{120}\right)=u(y) .
\end{aligned}
$$

This completes the proof.

Definition 7 [16] We define the space $W_{2}^{(3,3)}(\Omega)$ by

$$
W_{2}^{(3,3)}(\Omega)=\left\{\begin{array}{c}
u \mid \frac{\partial^{4} u}{\partial \eta^{2} \partial \tau^{2}} \text { is completely continuous in } \Omega=[0,1] \times[0, \tau], \\
\frac{\partial^{6} u}{\partial \eta^{3} \partial \tau^{3}} \in L^{2}(\Omega), u(\eta, 0)=0, \frac{\partial u(\eta, 0)}{\partial \tau}=0, u(0, \tau)=0, u(1, \tau)=0
\end{array}\right\}
$$

with the inner product and norm

$$
\begin{aligned}
\langle u, v\rangle_{W_{2}^{(3,3)}=} & \sum_{i=0}^{2} \int_{0}^{\tau}\left[\frac{\partial^{3}}{\partial \tau^{3}} \frac{\partial^{i}}{\partial \eta^{i}} u(0, \tau) \frac{\partial^{3}}{\partial \tau^{3}} \frac{\partial^{i}}{\partial \eta^{i}} v(0, \tau)\right] d \tau \\
& +\sum_{j=0}^{2}\left\langle\frac{\partial^{j}}{\partial \tau^{j}} u(\cdot, 0), \frac{\partial^{j}}{\partial \tau^{j}} v(\cdot, 0)\right\rangle_{W_{2}^{3}} \\
& +\int_{0}^{1} \int_{0}^{\tau}\left[\frac{\partial^{3}}{\partial \eta^{3}} \frac{\partial^{3}}{\partial \tau^{3}} u(\eta, \tau) \frac{\partial^{3}}{\partial \eta^{3}} \frac{\partial^{3}}{\partial \tau^{3}} v(\eta, \tau)\right] d \tau d \eta, \quad u, v \in W_{2}^{(3,3)}(\Omega)
\end{aligned}
$$


and

$$
\|u\|_{W}=\sqrt{\langle u, u\rangle_{W}}, \quad u \in W_{2}^{(3,3)}(\Omega) .
$$

Theorem 2.2 Let $K_{(y, s)}(\eta, \tau)$ be a reproducing kernel function $W_{2}^{(3,3)}(\Omega)$. We have

$$
K_{(y, s)}(\eta, \tau)=R_{y}(\eta) r_{s}(\tau)
$$

and for any $u \in W_{2}^{(3,3)}(\Omega)$,

$$
u(y, s)=\left\langle u(\eta, \tau), K_{(y, s)}(\eta, \tau)\right\rangle_{W_{2}^{(3,3)}}
$$

and

$$
K_{(y, s)}(\eta, \tau)=K_{(\eta, \tau)}(y, s)
$$

Definition 8 [16] We define the space $\widehat{W}_{2}^{(1,1)}(\Omega)$ by

$$
\widehat{W}_{2}^{(1,1)}(\Omega)=\left\{\begin{array}{c}
u \mid u \text { is completely continuos in } \Omega=[0,1] \times[0, \tau], \\
\frac{\partial^{2} u}{\partial \eta \partial \tau} \in L^{2}(\Omega)
\end{array}\right\}
$$

with the inner product and norm

$$
\begin{aligned}
\langle u, v\rangle_{\widehat{W}_{2}^{(1,1)}}= & \int_{0}^{\tau}\left[\frac{\partial}{\partial \tau} u(0, \tau) \frac{\partial}{\partial \tau} v(0, \tau)\right] d \tau+\langle u(\cdot, 0), v(\cdot, 0)\rangle_{G_{2}^{1}} \\
& +\int_{0}^{1} \int_{0}^{\tau}\left[\frac{\partial}{\partial \eta} \frac{\partial}{\partial \tau} u(\eta, \tau) \frac{\partial}{\partial \eta} \frac{\partial}{\partial \tau} v(\eta, \tau)\right] d \tau d \eta, \quad u, v \in \widehat{W}_{2}^{(1,1)}
\end{aligned}
$$

and

$$
\|u\|_{\widehat{W}_{2}^{(1,1)}}=\sqrt{\langle u, u\rangle_{\widehat{W}_{2}^{(1,1)}}}, \quad u \in \widehat{W}_{2}^{(1,1)} .
$$

$\widehat{W}_{2}^{(1,1)}(\Omega)$ is a reproducing kernel space, and its reproducing kernel function $G_{(y, s)}(\eta, \tau)$ is given as

$$
G_{(y, s)}(\eta, \tau)=Q_{y}(\eta) q_{s}(\tau)
$$

\section{Solutions in $W_{2}^{(3,3)}(\Omega)$}

In this section, we give the solution of (1)-(3) in the reproducing kernel space $W_{2}^{(3,3)}(\Omega)$. We define the linear operator $L: W_{2}^{(3,3)}(\Omega) \rightarrow \widehat{W}_{2}^{(1,1)}(\Omega)$ as

$$
L v=\frac{\partial^{2} v}{\partial \tau^{2}}(\eta, \tau)-\frac{\partial^{2} v}{\partial \eta^{2}}(\eta, \tau), \quad v \in W_{2}^{(3,3)}(\Omega) .
$$

If we homogenize the conditions of the model problem (1)-(3), then it changes to the following problem:

$$
\left\{\begin{array}{l}
L v=M(\eta, \tau), \quad(\eta, \tau) \in \Omega=[0,1] \times[0, \tau] \\
v(\eta, 0)=\frac{\partial v}{\partial \tau}(\eta, 0)=v(0, \tau)=v(1, \tau)=0
\end{array}\right.
$$


where

$$
\begin{aligned}
M(\eta, \tau)= & \frac{(\eta-1) f(\eta) h_{1}^{\prime \prime}(\tau)}{h_{1}(0)}-\eta h_{2}^{\prime \prime}(\tau)-\frac{2 f^{\prime}(\eta) h_{1}(\tau)}{h_{1}(0)}-\frac{(\eta-1) f^{\prime \prime}(\eta) h_{1}(\tau)}{h_{1}(0)} \\
& +2 f^{\prime}(\eta)+\eta f^{\prime \prime}(\eta)+\tau\left(g^{\prime \prime}(\eta)+\frac{2 g(0) f^{\prime}(\eta)}{h_{1}(0)}+\frac{(\eta-1) f^{\prime \prime}(\eta) g(0)}{h_{1}(0)}\right) \\
& -\sin \left[\begin{array}{c}
v(\eta, \tau)-\frac{(\eta-1) f(\eta) h_{1}(\tau)}{h_{1}(0)}+\eta h_{2}(\tau)+\eta f(\eta)-\eta h_{2}(0) \\
+\tau\left(g(\eta)+\frac{(\eta-1) f(\eta) g(0)}{h_{1}(0)}-\eta g(1)\right)
\end{array}\right]
\end{aligned}
$$

and

$$
\begin{aligned}
\nu(\eta, \tau)= & u(\eta, \tau)+\frac{(\eta-1) f(\eta) h_{1}(\tau)}{h_{1}(0)}-\eta h_{2}(\tau)-\eta f(\eta)+\eta h_{2}(0) \\
& -\tau\left(g(\eta)+\frac{(\eta-1) f(\eta) g(0)}{h_{1}(0)}-\eta g(1)\right)
\end{aligned}
$$

with $h_{1}(0) \neq 0$.

Lemma 3.1 The operator $L$ is bounded linear.

Proof By Definition 8 we have

$$
\begin{aligned}
\|L u\|_{\widehat{W}_{2}^{(1,1)}}^{2}= & \int_{0}^{\tau}\left[\frac{\partial}{\partial \tau} L u(0, \tau)\right]^{2} d \tau+\langle L u(\cdot, 0), L u(\cdot, 0)\rangle_{G_{2}^{1}} \\
& +\int_{0}^{1} \int_{0}^{\tau}\left[\frac{\partial}{\partial \eta} \frac{\partial}{\partial \tau} L u(\eta, \tau)\right]^{2} d \tau d \eta \\
= & \int_{0}^{\tau}\left[\frac{\partial}{\partial \tau} L u(0, \tau)\right]^{2} d \tau+[L u(0,0)]^{2} \\
& +\int_{0}^{1}\left[\frac{\partial}{\partial \eta} L u(\eta, 0)\right]^{2} d \eta+\int_{0}^{1} \int_{0}^{\tau}\left[\frac{\partial}{\partial \eta} \frac{\partial}{\partial \tau} L u(\eta, \tau)\right]^{2} d \tau d \eta .
\end{aligned}
$$

Since

$$
\begin{aligned}
& u(\eta, \tau)=\left\langle u(\xi, \gamma), K_{(\eta, \tau)}(\xi, \gamma)\right\rangle_{W_{2}^{(3,3)},}, \\
& L u(\eta, \tau)=\left\langle u(\xi, \gamma), L K_{(\eta, \tau)}(\xi, \gamma)\right\rangle_{W_{2}^{(3,3)},}
\end{aligned}
$$

from the continuity of $K_{(\eta, \tau)}(\xi, \gamma)$ we have

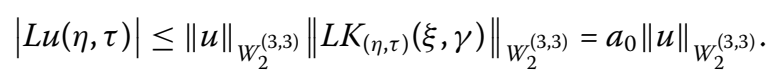

Accordingly, for $i=0,1$,

$$
\begin{aligned}
& \frac{\partial^{i}}{\partial \eta^{i}} L u(\eta, \tau)=\left\langle u(\xi, \gamma), \frac{\partial^{i}}{\partial \eta^{i}} L K_{(\eta, \tau)}(\xi, \gamma)\right\rangle_{W_{2}^{(3,3)}}, \\
& \frac{\partial}{\partial \tau} \frac{\partial^{i}}{\partial \eta^{i}} L u(\eta, \tau)=\left\langle u(\xi, \gamma), \frac{\partial}{\partial \tau} \frac{\partial^{i}}{\partial \eta^{i}} L K_{(\eta, \tau)}(\xi, \gamma)\right\rangle_{W_{2}^{(3,3)}},
\end{aligned}
$$


and then

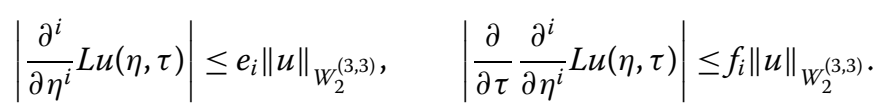

Therefore,

$$
\|L u(\eta, \tau)\|_{\widehat{W}_{2}^{(1,1)}}^{2} \leq \sum_{i=0}^{1}\left(e_{i}^{2}+\tau f_{i}^{2}\right)\|u\|_{W_{2}^{(3,3)}}^{2}=A\|u\|_{W_{2}^{(3,3)}}^{2},
$$

where $A=\sum_{i=0}^{1}\left(e_{i}^{2}+\tau f_{i}^{2}\right)$.

Now, choose a countable dense subset $\left\{\left(\eta_{1}, \tau_{1}\right),\left(\eta_{2}, \tau_{2}\right), \ldots\right\}$ in $\Omega$ and define

$$
\varphi_{i}(\eta, \tau)=G_{\left(\eta_{i}, \tau_{i}\right)}(\eta, \tau), \quad \Psi_{i}(\eta, \tau)=L^{*} \varphi_{i}(\eta, \tau)
$$

where $L^{*}$ is the adjoint operator of $L$. The orthonormal system $\left\{\widehat{\Psi}_{i}(\eta, \tau)\right\}_{i=1}^{\infty}$ of $W_{2}^{(3,3)}(\Omega)$ can be obtained by the Gram-Schmidt orthogonalization of $\left\{\Psi_{i}(\eta, \tau)\right\}_{i=1}^{\infty}$ as

$$
\widehat{\Psi}_{i}(\eta, \tau)=\sum_{k=1}^{i} \beta_{i k} \Psi_{k}(\eta, \tau) .
$$

Theorem 3.2 Assume that $\left\{\left(\eta_{i}, \tau_{i}\right)\right\}_{i=1}^{\infty}$ is dense in $\Omega$. Then $\left\{\Psi_{i}(\eta, \tau)\right\}_{i=1}^{\infty}$ is a complete system in $W_{2}^{(3,3)}(\Omega)$, and

$$
\Psi_{i}(\eta, \tau)=\left.L_{(y, s)} K_{(y, s)}(\eta, \tau)\right|_{(y, s)=\left(\eta_{i}, \tau_{i}\right)} .
$$

Proof We have

$$
\begin{aligned}
\Psi_{i}(\eta, \tau) & =\left(L^{*} \varphi_{i}\right)(\eta, \tau)=\left\langle\left(L^{*} \varphi_{i}\right)(y, s), K_{(\eta, \tau)}(y, s)\right\rangle_{W_{2}^{(3,3)}} \\
& =\left\langle\varphi_{i}(y, s),\left.L_{(y, s)} K_{(\eta, \tau)}(y, s)\right|_{\widehat{W}_{2}^{(1,1)}}\right. \\
& =\left.L_{(y, s)} K_{(\eta, \tau)}(y, s)\right|_{(y, s)=\left(\eta_{i}, \tau_{i}\right)} \\
& =\left.L_{(y, s)} K_{(y, s)}(\eta, \tau)\right|_{(y, s)=\left(\eta_{i}, \tau_{i}\right)} .
\end{aligned}
$$

Clearly, $\Psi_{i}(\eta, \tau) \in W(\Omega)$. For each fixed $u(\eta, \tau) \in W_{2}^{(3,3)}(\Omega)$, if

$$
\left\langle u(\eta, \tau),\left.\Psi_{i}(\eta, \tau)\right|_{W_{2}^{(3,3)}}=0, \quad i=1,2, \ldots,\right.
$$

then

$$
\left\langle u(\eta, \tau),\left.\left(L^{*} \varphi_{i}\right)(\eta, \tau)\right|_{W_{2}^{(3,3)}}=\left\langle L u(\eta, \tau),\left.\varphi_{i}(\eta, \tau)\right|_{\widehat{W}_{2}^{(1,1)}}=(L u)\left(\eta_{i}, \tau_{i}\right)=0, \quad i=1,2, \ldots\right.\right.
$$

Since $\left\{\left(\eta_{i}, \tau_{i}\right)\right\}_{i=1}^{\infty}$ is dense in $\Omega,(L u)(\eta, \tau)=0$. Therefore, $u=0$ by the existence of $L^{-1}$.

Theorem 3.3 If $\left\{\left(\eta_{i}, \tau_{i}\right)\right\}_{i=1}^{\infty}$ is dense in $\Omega$, then the solution of (5) is

$$
u=\sum_{i=1}^{\infty} \sum_{k=1}^{i} \beta_{i k} M\left(\eta_{k}, \tau_{k}\right) \widehat{\Psi}_{i}(\eta, \tau) .
$$


Proof The system $\left\{\Psi_{i}(\eta, \tau)\right\}_{i=1}^{\infty}$ is complete in $W_{2}^{(3,3)}(\Omega)$. Therefore, we get

$$
\begin{aligned}
u & =\sum_{i=1}^{\infty}\left\langle u, \widehat{\Psi}_{i}\right\rangle_{W_{2}^{(3,3)}} \widehat{\Psi}_{i}=\sum_{i=1}^{\infty} \sum_{k=1}^{i} \beta_{i k}\left\langle u, \Psi_{k}\right\rangle_{W_{2}^{(3,3)}} \widehat{\Psi}_{i}=\sum_{i=1}^{\infty} \sum_{k=1}^{i} \beta_{i k}\left\langle u, L^{*} \varphi_{k}\right\rangle_{W_{2}^{(3,3)}} \widehat{\Psi}_{i} \\
& =\sum_{i=1}^{\infty} \sum_{k=1}^{i} \beta_{i k}\left\langle L u, \varphi_{k}\right\rangle_{\widehat{W}_{2}^{(1,1)}} \widehat{\Psi}_{i}=\sum_{i=1}^{\infty} \sum_{k=1}^{i} \beta_{i k}\left\langle L u, G_{\left(\eta_{k}, \tau_{k}\right)}\right\rangle_{\widehat{W}_{2}^{(1,1)}} \widehat{\Psi}_{i} \\
& =\sum_{i=1}^{\infty} \sum_{k=1}^{i} \beta_{i k} L u\left(\eta_{k}, \tau_{k}\right) \widehat{\Psi}_{i}=\sum_{i=1}^{\infty} \sum_{k=1}^{i} \beta_{i k} M\left(\eta_{k}, \tau_{k}\right) \widehat{\Psi}_{i} .
\end{aligned}
$$

This completes the proof.

Now an approximate solution $u_{n}$ can be obtained from the $n$-term intercept of the exact solution $u$ :

$$
u_{n}=\sum_{i=1}^{n} \sum_{k=1}^{i} \beta_{i k} M\left(\eta_{k}, \tau_{k}\right) \widehat{\Psi}_{i}(\eta, \tau) .
$$

Obviously,

$$
\left\|u_{n}(\eta, \tau)-u(\eta, \tau)\right\| \rightarrow 0 \quad(n \rightarrow \infty)
$$

\section{The method implementation}

If $M$ is linear, then the analytical solution of (5) can be obtained directly by (6). If $M$ is nonlinear, then the solution of (5) can be obtained either by (6) or by an iterative method as follows. We construct an iterative sequence $u_{n}$ by putting

$$
\left\{\begin{array}{l}
\text { any fixed } u_{0} \in W_{2}^{(3,3)}, \\
u_{n}=\sum_{i=1}^{n} A_{i} \widehat{\Psi}_{i}
\end{array}\right.
$$

where

$$
\left\{\begin{array}{l}
A_{1}=\beta_{11} M\left(\eta_{k}, \tau_{k}\right), \\
A_{2}=\sum_{k=1}^{2} \beta_{2 k} M\left(\eta_{k}, \tau_{k}\right), \\
\cdots \\
A_{n}=\sum_{k=1}^{n} \beta_{n k} M\left(\eta_{k}, \tau_{k}\right) .
\end{array}\right.
$$

Lemma 4.1 If

$u_{n} \stackrel{\|\cdot\|}{\rightarrow} \widehat{u}, \quad\left\|u_{n}\right\|$ is bounded, $\left(\eta_{n}, \tau_{n}\right) \rightarrow(y, s)$, and $M(\eta, \tau)$ is continuous

then

$$
M\left(\eta_{n}, \tau_{n}\right) \rightarrow M(y, s)
$$

Proof By the reproducing property and Cauchy-Schwarz inequality we have

$$
\begin{aligned}
|u(\eta, \tau)| & =\mid\left\langle u(y, s),\left.K_{(\eta, \tau)}(y, s)\right|_{W_{2}^{(3,3)}}\right| \\
& \leq\|u(y, s)\|_{W_{2}^{(3,3)}}\left\|K_{(\eta, \tau)}(y, s)\right\|_{W_{2}^{(3,3)}}=N_{1}\|u(y, s)\|_{W_{2}^{(3,3)}} .
\end{aligned}
$$


Thus, we obtain

$$
\begin{aligned}
\left|\frac{\partial u(\eta, \tau)}{\partial \eta}\right| & =\left|\left\langle u(y, s), \frac{\partial K_{(\eta, \tau)}(y, s)}{\partial \eta}\right\rangle_{W_{2}^{(3,3)}}\right| \leq\|u(y, s)\|_{W_{2}^{(3,3)}}\left\|\frac{\partial K_{(\eta, \tau)}(y, s)}{\partial \eta}\right\|_{W_{2}^{(3,3)}} \\
& =N_{2}\|u(y, s)\|_{W_{2}^{(3,3)}}
\end{aligned}
$$

and

$$
\begin{aligned}
\left|\frac{\partial u(\eta, \tau)}{\partial \tau}\right| & =\left|\left\langle u(y, s), \frac{\partial K_{(\eta, \tau)}(y, s)}{\partial \tau}\right\rangle_{W_{2}^{(3,3)}}\right| \leq\|u(y, s)\|_{W}\left\|\frac{\partial K_{(\eta, \tau)}(y, s)}{\partial \tau}\right\|_{W_{2}^{(3,3)}} \\
& =N_{3}\|u(y, s)\|_{W_{2}^{(3,3)}}
\end{aligned}
$$

One the one hand, we have

$$
\begin{aligned}
\left|u_{n-1}(y, s)-\widehat{u}(y, s)\right| & =\left|\left\langle u_{n-1}(\eta, \tau)-\widehat{u}(\eta, \tau), K_{(y, s)}(\eta, \tau)\right\rangle_{W_{2}^{(3,3)}}\right| \\
& \leq\left\|u_{n-1}(\eta, \tau)-\widehat{u}(\eta, \tau)\right\|_{W_{2}^{(3,3)}}\left\|K_{(\eta, \tau)}(y, s)\right\|_{W_{2}^{(3,3)}} \\
& =N_{4}\left\|u_{n-1}(\eta, \tau)-\widehat{u}(\eta, \tau)\right\|_{W_{2}^{(3,3)}}
\end{aligned}
$$

on the other hand, we get

$$
\begin{aligned}
\left|u_{n-1}\left(\eta_{n}, \tau_{n}\right)-\widehat{u}(y, s)\right| & =\left|u_{n-1}\left(\eta_{n}, \tau_{n}\right)-u_{n-1}(y, s)+u_{n-1}(y, s)-\widehat{u}(y, s)\right| \\
& \leq\left|\nabla u_{n-1}(\xi, \eta)\right|\left|\left(\eta_{n}, \tau_{n}\right)-(y, s)\right|+\left|u_{n-1}(y, s)-\widehat{u}(y, s)\right| .
\end{aligned}
$$

Using these inequalities with $u_{n} \stackrel{\|\cdot\|}{\rightarrow} \widehat{u}$, we find

$$
\left|u_{n-1}(y, s)-\widehat{u}(y, s)\right| \rightarrow 0, \quad\left|\nabla u_{n-1}(\xi, \eta)\right| \leq \sqrt{c_{1}^{2}+c_{2}^{2}}\|u\|_{W_{2}^{(3,3)}}
$$

Therefore, as $n \rightarrow \infty$, using the boundedness of $\left\|u_{n}\right\|$ gives

$$
\left|u_{n-1}\left(\eta_{n}, \tau_{n}\right)-\widehat{u}(y, s)\right| \rightarrow 0
$$

As $n \rightarrow \infty$, with the continuity of $M(\eta, \tau)$ we get

$$
M\left(\eta_{n}, \tau_{n}\right) \rightarrow M(y, s)
$$

This completes the proof.

Theorem 4.2 Assume that $\left\|u_{n}\right\|$ is a bounded in (7) and that (5) has a unique solution. If $\left\{\left(\eta_{i}, \tau_{i}\right)\right\}_{i=1}^{\infty}$ is dense in $W_{2}^{(3,3)}(\Omega)$, then the $n$-term approximate solutions $u_{n}(\eta, \tau)$ converge to the analytical solution $u(\eta, \tau)$ of $(5)$, and

$$
u(\eta, \tau)=\sum_{i=1}^{\infty} A_{i} \widehat{\Psi}_{i}(\eta, \tau)
$$

where $A_{i}$ is given by (8). 
Proof First, we prove the convergence of $u_{n}(\eta, \tau)$. From (7) we infer that

$$
u_{n+1}(\eta, \tau)=u_{n}(\eta, \tau)+A_{n+1} \widehat{\Psi}_{n+1}(\eta, \tau)
$$

The orthonormality of $\left\{\widehat{\Psi}_{i}\right\}_{i=1}^{\infty}$ yields that

$$
\left\|u_{n+1}\right\|^{2}=\left\|u_{n}\right\|^{2}+A_{n+1}^{2}=\sum_{i=1}^{n+1} A_{i}^{2} .
$$

In terms of (9), we have that $\left\|u_{n+1}\right\|>\left\|u_{n}\right\|$. Since $\left\|u_{n}\right\|$ is bounded, $\left\|u_{n}\right\|$ is convergent, and there exists a constant $c$ such that

$$
\sum_{i=1}^{\infty} A_{i}^{2}=c
$$

This implies that

$$
\left\{A_{i}\right\}_{i=1}^{\infty} \in l^{2}
$$

If $m>n$, then

$$
\begin{aligned}
\left\|u_{m}-u_{n}\right\|^{2} & =\left\|u_{m}-u_{m-1}+u_{m-1}-u_{m-2}+\cdots+u_{n+1}-u_{n}\right\|^{2} \\
& \leq\left\|u_{m}-u_{m-1}\right\|^{2}+\left\|u_{m-1}-u_{m-2}\right\|^{2}+\cdots+\left\|u_{n+1}-u_{n}\right\|^{2} .
\end{aligned}
$$

Since

$$
\left\|u_{m}-u_{m-1}\right\|^{2}=A_{m}^{2}
$$

we have

$$
\left\|u_{m}-u_{n}\right\|^{2}=\sum_{l=n+1}^{m} A_{l}^{2} \rightarrow 0 \quad \text { as } n \rightarrow \infty .
$$

The completeness of $W_{2}^{(3,3)}(\Omega)$ shows that $u_{n} \rightarrow \widehat{u}$ as $n \rightarrow \infty$. We have

$$
\widehat{u}(\eta, \tau)=\sum_{i=1}^{\infty} A_{i} \widehat{\Psi}_{i}(\eta, \tau) .
$$

Note that

$$
(L \widehat{u})(\eta, \tau)=\sum_{i=1}^{\infty} A_{i} L \widehat{\Psi}_{i}(\eta, \tau)
$$

and

$$
\begin{aligned}
(L \widehat{u})\left(\eta_{l}, \tau_{l}\right) & =\sum_{i=1}^{\infty} A_{i} L \widehat{\Psi}_{i}\left(\eta_{l}, \tau_{l}\right)=\sum_{i=1}^{\infty} A_{i}\left\langle L \widehat{\Psi}_{i}(\eta, \tau),\left.\varphi_{l}(\eta, \tau)\right|_{\widehat{W}_{2}^{(1,1)}}\right. \\
& =\sum_{i=1}^{\infty} A_{i}\left\langle\widehat{\Psi}_{i}(\eta, \tau), L^{*} \varphi_{l}(\eta, \tau)\right\rangle_{W_{2}^{(3,3)}}=\sum_{i=1}^{\infty} A_{i}\left\langle\widehat{\Psi}_{i}(\eta, \tau), \Psi_{l}(\eta, \tau)\right\rangle_{W_{2}^{(3,3)}}
\end{aligned}
$$


Therefore,

$$
\begin{aligned}
\sum_{l=1}^{i} \beta_{i l}(L \widehat{u})\left(\eta_{l}, \tau_{l}\right) & =\sum_{i=1}^{\infty} B_{i}\left\langle\widehat{\Psi}_{i}(\eta, \tau), \sum_{l=1}^{i} \beta_{i l} \Psi_{l}(\eta, \tau)\right\rangle_{W_{2}^{(3,3)}} \\
& =\sum_{i=1}^{\infty} B_{i}\left\langle\widehat{\Psi}_{i}(\eta, \tau), \widehat{\Psi}_{l}(\eta, \tau)\right\rangle_{W_{2}^{(3,3)}}=A_{l} .
\end{aligned}
$$

In view of (8), we have

$$
L \widehat{u}\left(\eta_{l}, \tau_{l}\right)=M\left(\eta_{l}, \tau_{l}\right) .
$$

Since $\left\{\left(\eta_{i}, \tau_{i}\right)\right\}_{i=1}^{\infty}$ is dense in $\Omega$, for each $(y, s) \in \Omega$, there exists a subsequence $\left\{\left(\eta_{n_{j}}, \tau_{n_{j}}\right)\right\}_{j=1}^{\infty}$ such that

$$
\left(\eta_{n_{j}}, \tau_{n_{j}}\right) \rightarrow(y, s) \quad(j \rightarrow \infty)
$$

We know that

$$
L \widehat{u}\left(\eta_{n_{j}}, \tau_{n_{j}}\right)=M\left(\eta_{n_{j}}, \tau_{n_{j}}\right) .
$$

Let $j \rightarrow \infty$; by the continuity of $M$ we have

$$
(L \widehat{u})(y, s)=M(y, s),
$$

which proves that $\widehat{u}(\eta, \tau)$ satisfies (5).

We obtain an approximate solution $\zeta_{n}(t)$ as

$$
\zeta_{n}(t)=\sum_{i=1}^{n} \sum_{k=1}^{i} \sigma_{i k} z\left(t_{k}, \zeta\left(t_{k}\right)\right) \widehat{\eta}_{i}(t)
$$

Remark Let consider a countable dense set

$$
\left\{\left(\eta_{1}, \tau_{1}\right),\left(\eta_{2}, \tau_{2}\right), \ldots\right\} \in \Omega
$$

and define

$$
\varphi_{i}=G_{\left(\eta_{i}, \tau_{i}\right)}, \quad \Psi_{i}=L^{*} \varphi_{i}, \quad \widehat{\Psi}_{i}=\sum_{k=1}^{i} \beta_{i k} \Psi_{k}
$$

Then the coefficients $\beta_{i k}$ can be found by

$$
\beta_{11}=\frac{1}{\left\|\Psi_{1}\right\|}, \quad \beta_{i i}=\frac{1}{\sqrt{\left\|\Psi_{i}\right\|^{2}}-\sum_{k=1}^{i-1} c_{i k}^{2}}, \quad \beta_{i j}=\frac{-\sum_{k=j}^{i-1} c_{i k} \beta_{k j}}{\sqrt{\left\|\Psi_{i}\right\|^{2}}-\sum_{k=1}^{i-1} c_{i k}^{2}}, \quad c_{i k}=\left\langle\Psi_{i}, \widehat{\Psi}_{k}\right\rangle .
$$




\section{Numerical experiments}

In this section, we solve two examples were solved with RKM. We show our results by tables and figures. The numerical results are compared with exact solutions and existing numerical approximations to illustrate the efficiency and high accuracy of the method. The method presents the solutions in terms of convergent series with easily computable components and improves the convergence of the series solution. The method was used

Table 1 Numerical results for Example 5.1

\begin{tabular}{llllll}
\hline $\boldsymbol{\eta}$ & $\boldsymbol{\tau}$ & ES & AS & AE & $\begin{array}{l}\text { Time } \\
\text { CPU (s) }\end{array}$ \\
\hline 0.1 & 0.1 & 0.2938926262 & 0.2938930965 & $4.703 \times 10^{-7}$ & 3.860 \\
0.2 & 0.2 & 0.4755282582 & 0.4755313577 & $3.0995 \times 10^{-6}$ & 3.016 \\
0.3 & 0.3 & 0.4755282582 & 0.4755183355 & $9.9227 \times 10^{-6}$ & 2.984 \\
0.4 & 0.4 & 0.2938926261 & 0.2939007109 & $8.0848 \times 10^{-6}$ & 3.000 \\
0.5 & 0.5 & 0.0 & 0.0000282140 & $2.82140 \times 10^{-5}$ & 3.094 \\
0.6 & 0.6 & -0.2938926264 & -0.2939063137 & $1.36873 \times 10^{-5}$ & 3.031 \\
0.7 & 0.7 & -0.4755282583 & -0.4755305759 & $2.3176 \times 10^{-6}$ & 3.031 \\
0.8 & 0.8 & -0.4755282581 & -0.4755277748 & $4.833 \times 10^{-7}$ & 2.953 \\
0.9 & 0.9 & -0.2938926260 & -0.2938966580 & $4.0320 \times 10^{-6}$ & 3.204 \\
1.0 & 1.0 & 0.0 & $-3.690702068 \times 10^{-7}$ & $3.690702068 \times 10^{-7}$ & 3.578 \\
\hline
\end{tabular}

Table 2 Numerical results for Example 5.1 with $\tau=1$

\begin{tabular}{lll}
\hline $\boldsymbol{\eta}$ & ES & AE \\
& AS & RE \\
\hline-0.80 & 0.5877852522 & $1.756 \times 10^{-7}$ \\
& 0.5877854278 & $2.987485639 \times 10^{-7}$ \\
-0.40 & 0.9510565165 & $7.70 \times 10^{-8}$ \\
& 0.9510565935 & $8.096259125 \times 10^{-8}$ \\
0.40 & -0.9510565165 & $7.70 \times 10^{-8}$ \\
& -0.9510565935 & $8.096259125 \times 10^{-8}$ \\
0.80 & -0.5877852522 & $1.756 \times 10^{-7}$ \\
& -0.5877854278 & $2.987485639 \times 10^{-7}$ \\
\hline
\end{tabular}

Table 3 Comparison of AE and RE for Example 5.1

\begin{tabular}{lllll}
\hline $\boldsymbol{\eta}$ & AE [27] & AE [RKM] & RE [27] & RE [RKM] \\
\hline-0.80 & $1.94 E-05$ & $1.756 E-07$ & $3.29 E-05$ & $2.987485639 E-7$ \\
-0.40 & $2.84 E-07$ & $7.700 E-08$ & $2.98 E-07$ & $8.096259125 E-8$ \\
0.40 & $2.84 E-07$ & $7.700 E-08$ & $2.98 E-07$ & $8.096259125 E-8$ \\
0.80 & $1.94 E-05$ & $1.756 E-07$ & $3.29 E-05$ & $2.987485639 E-7$ \\
\hline
\end{tabular}

Figure 1 Plots of RKM solution for Example 5.1.

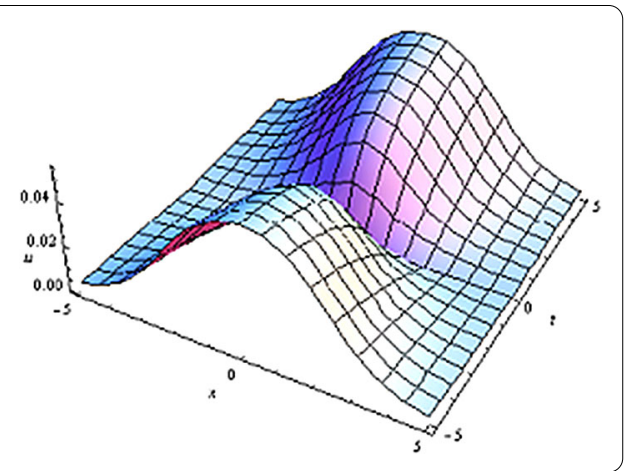


Figure 2 Plots of absolute error for Example 5.1.

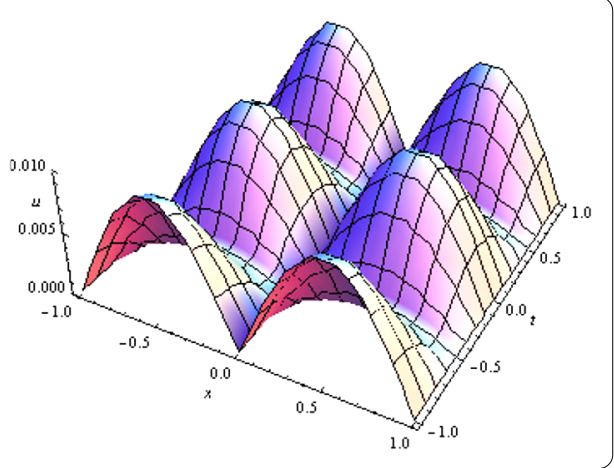

Figure 3 Plots of absolute error for Example 5.1.
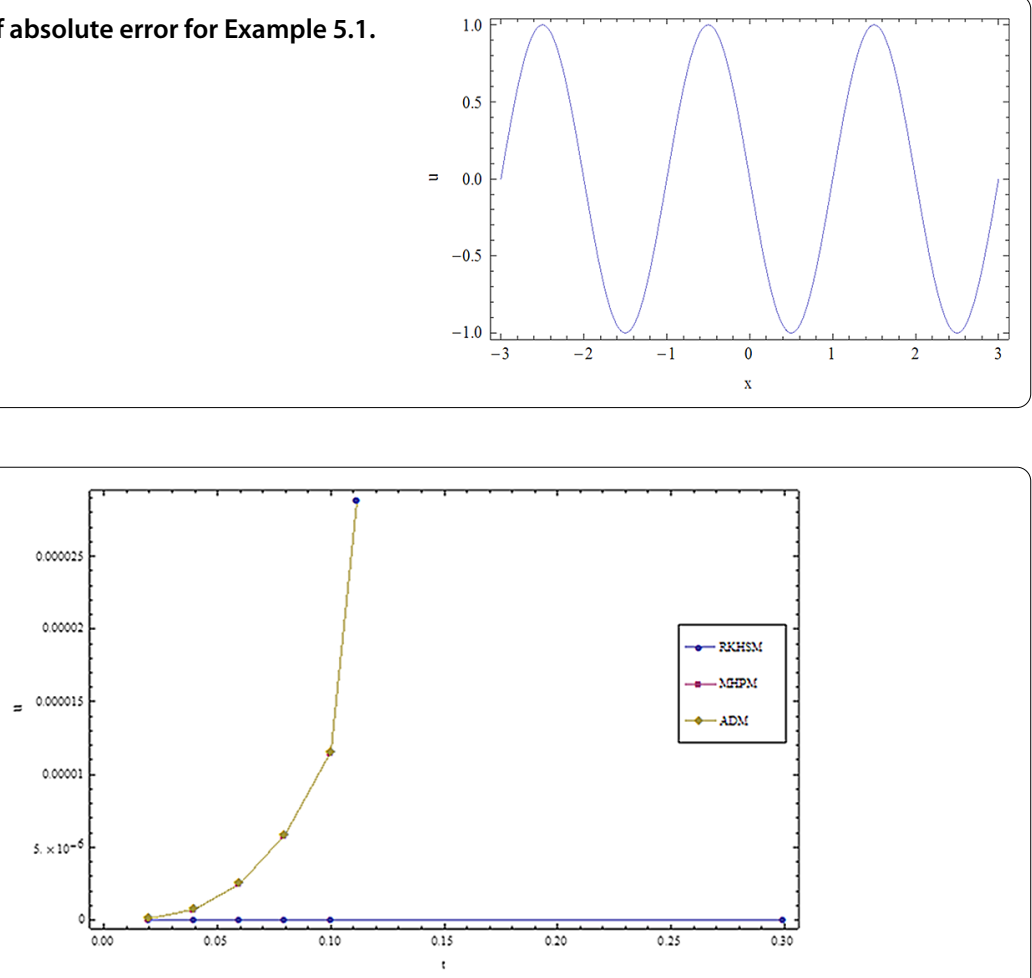

Figure 4 Plots of absolute error for Example 5.1.

in a direct way without using restrictive assumptions. Throughout this work, all computations are implemented by using Maple 16 software package.

Example 5.1 Let us consider the problem with the following initial conditions:

$$
u(\eta, 0)=\sin (\pi \eta), \quad \frac{\partial u}{\partial \tau}(\eta, 0)=0 .
$$

The exact solution is [28]

$$
u(\eta, \tau)=\frac{1}{2}(\sin \pi(\eta+\tau)+\sin \pi(\eta-\tau)) .
$$

After homogenizing the initial conditions and using our method, we obtain the results presented in Tables 1-3 and Figures 1-4. 
Table 4 Numerical results for Example 5.2

\begin{tabular}{lllllll}
\hline $\boldsymbol{\eta}$ & $\boldsymbol{\tau}$ & ES & AS & AE & RE & $\begin{array}{l}\text { Time } \\
\text { CPU (s) }\end{array}$ \\
\hline 0.1 & 0.1 & 0.396702532289366215 & 0.39670253228936612 & $9.43 \times 10^{-17}$ & $2.37 \times 10^{-16}$ & 0.874 \\
0.2 & 0.2 & 0.77443854423966038 & 0.774438544239660056 & $3.24 \times 10^{-16}$ & $4.19 \times 10^{-16}$ & 0.843 \\
0.3 & 0.3 & 1.11790876976715877 & 1.117908769767159201 & $4.29 \times 10^{-16}$ & $3.83 \times 10^{-16}$ & 0.874 \\
0.4 & 0.4 & 1.41753016381685945 & 1.417530163816859722 & $2.65 \times 10^{-16}$ & $1.87 \times 10^{-16}$ & 0.952 \\
0.5 & 0.5 & 1.66943886908587781 & 1.669438869085877479 & $3.33 \times 10^{-16}$ & $1.99 \times 10^{-16}$ & 0.874 \\
0.6 & 0.6 & 1.87415961287513759 & 1.874159612875137133 & $4.57 \times 10^{-16}$ & $2.44 \times 10^{-16}$ & 0.904 \\
0.7 & 0.7 & 2.03492391748435838 & 2.034923917484357432 & $9.47 \times 10^{-16}$ & $4.65 \times 10^{-16}$ & 0.921 \\
0.8 & 0.8 & 2.15626165034295019 & 2.156261650342951155 & $9.63 \times 10^{-16}$ & $4.46 \times 10^{-16}$ & 0.967 \\
0.9 & 0.9 & 2.24305837947587106 & 2.243058379475871257 & $1.88 \times 10^{-16}$ & $8.39 \times 10^{-17}$ & 0.999 \\
1.0 & 1.0 & 2.30002473031364741 & 2.300024730313647325 & $8.66 \times 10^{-17}$ & $3.76 \times 10^{-17}$ & 0.967 \\
\hline
\end{tabular}

Table 5 Numerical results for Example 5.2 with $\tau=1$

\begin{tabular}{rlllll}
\hline $\boldsymbol{\eta}$ & ES & AS & AE & RE & $\begin{array}{l}\text { Time } \\
\text { CPU (s) }\end{array}$ \\
\hline-0.80 & 2.5681097221289163512 & 2.5681097220865804301 & $4.2 \times 10^{-11}$ & $1.6 \times 10^{-11}$ & 0.842 \\
-0.40 & 2.9858433445292456583 & 2.9858433445285564413 & $6.8 \times 10^{-13}$ & $2.3 \times 10^{-13}$ & 0.873 \\
0.00 & 3.1415926535897932385 & 3.1415926535905335235 & $7.4 \times 10^{-13}$ & $2.3 \times 10^{-13}$ & 0.936 \\
0.40 & 2.9858433445292456583 & 2.9858433445285564413 & $6.8 \times 10^{-13}$ & $2.3 \times 10^{-13}$ & 0.686 \\
0.80 & 2.5681097221289163512 & 2.5681097220865804301 & $4.2 \times 10^{-11}$ & $1.6 \times 10^{-11}$ & 0.733 \\
\hline
\end{tabular}

Table 6 Comparison of absolute and relative errors for Example 5.2

\begin{tabular}{lllll}
\hline $\boldsymbol{\eta}$ & $\begin{array}{l}\text { AE } \\
\text { [27] }\end{array}$ & $\begin{array}{l}\text { AE } \\
\text { [RKM] }\end{array}$ & $\begin{array}{l}\text { RE } \\
\text { [27] }\end{array}$ & $\begin{array}{l}\text { RE } \\
\text { [RKM] }\end{array}$ \\
\hline-0.80 & $1.53 E-08$ & $4.23359211 E-11$ & $5.96 E-09$ & $1.64852462241777932 E-11$ \\
-0.40 & $3.54 E-10$ & $6.89217 E-13$ & $1.18 E-10$ & $2.30828252012217808 E-13$ \\
0.00 & $1.62 E-10$ & $7.40285 E-13$ & $5.15 E-11$ & $2.35640034093567477 E-13$ \\
0.40 & $3.54 E-10$ & $6.89217 E-13$ & $1.18 E-10$ & $2.30828252012217808 E-13$ \\
0.80 & $1.53 E-08$ & $4.23359211 E-11$ & $5.96 E-09$ & $1.64852462241777932 E-11$ \\
\hline
\end{tabular}

Table 7 Numerical results for Example 5.2 with $\eta=2.5$

\begin{tabular}{llllll}
\hline $\boldsymbol{\tau}$ & ES & AS & AE & RE & $\begin{array}{l}\text { Time } \\
\text { CPU (s) }\end{array}$ \\
\hline 0.02 & 0.013045652299470337726 & 0.013045652299470429228 & $9.15 \times 10^{-17}$ & $7.01 \times 10^{-15}$ & 1.014 \\
0.04 & 0.026091027076458045383 & 0.026091027076458055762 & $1.03 \times 10^{-17}$ & $3.97 \times 10^{-16}$ & 0.905 \\
0.06 & 0.039135846843901571207 & 0.039135846843901591760 & $2 . \times 10^{-17}$ & $5.25 \times 10^{-16}$ & 0.873 \\
0.08 & 0.05217983418557021854 & 0.052179834185570326022 & $1.07 \times 10^{-16}$ & $2.05 \times 10^{-15}$ & 0.842 \\
0.1 & 0.065222711791451326376 & 0.06522271179145110938 & $2.16 \times 10^{-16}$ & $3.32 \times 10^{-15}$ & 0.858 \\
0.3 & 0.19552959072837645953 & 0.19552959072837616718 & $2.9 \times 10^{-16}$ & $1.49 \times 10^{-15}$ & 0.749 \\
\hline
\end{tabular}

Table 8 Numerical solutions for Example 5.2 with $\eta=5.0$

\begin{tabular}{llllll}
\hline $\boldsymbol{\tau}$ & ES & AS & AE & RE & $\begin{array}{l}\text { Time } \\
\text { CPU (s) }\end{array}$ \\
\hline 0.02 & 0.0010780225516042560299 & 0.0010780225516046950169 & $4.3 \times 10^{-16}$ & $4.07 \times 10^{-13}$ & 1.233 \\
0.04 & 0.0021560449466078880312 & 0.0021560449466103154491 & $2.4 \times 10^{-15}$ & $1.12 \times 10^{-12}$ & 0.655 \\
0.06 & 0.003234067028410408468 & 0.0032340670284064571408 & $3.9 \times 10^{-15}$ & $1.22 \times 10^{-12}$ & 0.811 \\
0.08 & 0.0043120886404116027904 & 0.0043120886404125186434 & $9.1 \times 10^{-16}$ & $2.12 \times 10^{-13}$ & 0.936 \\
0.1 & 0.0053901096260116659256 & 0.0053901096260170153463 & $5.3 \times 10^{-15}$ & $9.92 \times 10^{-13}$ & 1.092 \\
0.3 & 0.016170250578558993341 & 0.016170250578577492240 & $1.8 \times 10^{-14}$ & $1.14 \times 10^{-12}$ & 1.139 \\
\hline
\end{tabular}


Table 9 Numerical results for Example 5.2 with $\eta=7.5$

\begin{tabular}{llllll}
\hline $\boldsymbol{\tau}$ & ES & AS & AE & RE & $\begin{array}{l}\text { Time } \\
\text { CPU (s) }\end{array}$ \\
\hline 0.02 & 0.0000884934721388573766 & 0.0000884934721392853003 & $4.2 \times 10^{-16}$ & $4.8 \times 10^{-12}$ & 0.827 \\
0.04 & 0.0001769869441910896592 & 0.0001769869441950110056 & $3.9 \times 10^{-15}$ & $2.2 \times 10^{-11}$ & 0.749 \\
0.06 & 0.0002654804160700717544 & 0.0002654804160796786727 & $9.6 \times 10^{-15}$ & $3.6 \times 10^{-11}$ & 0.889 \\
0.08 & 0.0003539738876891785695 & 0.0003539738876799533295 & $9.2 \times 10^{-15}$ & $2.6 \times 10^{-11}$ & 0.967 \\
0.1 & 0.0004424673589617850136 & 0.0004424673589703796322 & $8.5 \times 10^{-15}$ & $1.9 \times 10^{-11}$ & 0.812 \\
0.3 & 0.0013274020335728111501 & 0.0013274020333220852196 & $2.5 \times 10^{-13}$ & $1.8 \times 10^{-10}$ & 1.326 \\
\hline
\end{tabular}

Table 10 Numerical results for Example 5.2 with $\eta=10.0$

\begin{tabular}{llllll}
\hline $\boldsymbol{\tau}$ & ES & AS & AE & RE & $\begin{array}{l}\text { Time } \\
\text { CPU (s) }\end{array}$ \\
\hline 0.02 & 0.00000726398874701739435 & 0.0000072639887470122372 & $5.1 \times 10^{-18}$ & $7.0 \times 10^{-13}$ & 0.874 \\
0.04 & 0.00001452797749398687768 & 0.0000145279774939233104 & $6.3 \times 10^{-17}$ & $4.3 \times 10^{-12}$ & 0.936 \\
0.06 & 0.00002179196624086053894 & 0.0000217919662408353413 & $2.5 \times 10^{-17}$ & $1.1 \times 10^{-12}$ & 0.998 \\
0.08 & 0.00002905595498759046712 & 0.0000290559549867947262 & $7.9 \times 10^{-16}$ & $2.7 \times 10^{-11}$ & 1.170 \\
0.1 & 0.00003631994373412875118 & 0.000036319943734399877 & $2.7 \times 10^{-16}$ & $7.4 \times 10^{-12}$ & 0.858 \\
0.3 & 0.00010895983117843073892 & 0.0001089598311794268356 & $9.9 \times 10^{-16}$ & $9.1 \times 10^{-12}$ & 0.889 \\
\hline
\end{tabular}

Table 11 Comparison of absolute errors for Example 5.2 with $\eta=2.5$ and $\eta=5.0$

\begin{tabular}{lllllll}
\hline $\boldsymbol{\tau}$ & AE & AE & AE & AE & AE & AE \\
& RKM & MHPM & ADM & RKM & MHPM & ADM \\
& $\boldsymbol{\eta}=\mathbf{2 . 5}$ & $\boldsymbol{\eta}=\mathbf{2 . 5}[\mathbf{2 9}]$ & $\boldsymbol{\eta}=\mathbf{2 . 5}[\mathbf{2 9}]$ & $\boldsymbol{\eta}=\mathbf{5 . 0}$ & $\boldsymbol{\eta}=\mathbf{5 . 0}[\mathbf{2 9}]$ & $\boldsymbol{\eta}=\mathbf{5 . 0}[\mathbf{2 9}]$ \\
\hline 0.02 & $9.15 E-17$ & $9.25104 E-8$ & $9.25104 E-8$ & $4.3 E-16$ & $5.22002 E-11$ & $5.22002 E-11$ \\
0.04 & $1.03 E-17$ & $7.40084 E-7$ & $7.40084 E-7$ & $2.4 E-15$ & $4.17602 E-10$ & $4.17602 E-10$ \\
0.06 & $2 . E-17$ & $2.49778 E-6$ & $2.49778 E-6$ & $3.9 E-15$ & $1.40941 E-9$ & $1.40941 E-9$ \\
0.08 & $1.07 E-16$ & $5.92068 E-6$ & $5.92068 E-6$ & $9.1 E-16$ & $3.34082 E-9$ & $3.34082 E-9$ \\
0.1 & $2.16 E-16$ & $1.15638 E-5$ & $1.15638 E-5$ & $5.3 E-15$ & $6.52506 E-9$ & $6.52506 E-9$ \\
0.3 & $2.9 E-16$ & $3.12304 E-4$ & $3.12304 E-4$ & $1.8 E-14$ & $1.76230 E-7$ & $1.76230 E-7$ \\
\hline
\end{tabular}

Table 12 Comparison of absolute errors for Example 5.2 with $\eta=7.5$ and $\eta=10.0$

\begin{tabular}{lllllll}
\hline $\boldsymbol{\tau}$ & AE & AE & AE & AE & AE & AE \\
& RKM & MHPM & ADM & RKM & MHPM & ADM \\
& $\boldsymbol{\eta}=\mathbf{7 . 5}$ & $\boldsymbol{\eta}=\mathbf{7 . 5}[\mathbf{2 9}]$ & $\boldsymbol{\eta}=\mathbf{7 . 5}[\mathbf{2 9}]$ & $\boldsymbol{\eta}=\mathbf{1 0 . 0}$ & $\boldsymbol{\eta}=\mathbf{1 0 . 0}[\mathbf{2 9}]$ & $\boldsymbol{\eta}=\mathbf{1 0 . 0}[\mathbf{2 9}]$ \\
\hline 0.02 & $4.2 E-16$ & $2.88750 E-14$ & $2.88750 E-14$ & $5.1 E-18$ & $1.59700 E-17$ & $1.59700 E-17$ \\
0.04 & $3.9 E-15$ & $2.31000 E-13$ & $2.31000 E-13$ & $6.3 E-17$ & $1.27763 E-16$ & $1.27763 E-16$ \\
0.06 & $9.6 E-15$ & $7.79626 E-13$ & $7.79626 E-13$ & $2.5 E-17$ & $4.31201 E-16$ & $4.31201 E-16$ \\
0.08 & $9.2 E-15$ & $1.84800 E-12$ & $1.84800 E-12$ & $7.9 E-16$ & $1.02210 E-15$ & $1.02210 E-15$ \\
0.1 & $8.5 E-15$ & $3.60939 E-12$ & $3.60939 E-12$ & $2.7 E-16$ & $1.99629 E-15$ & $1.99629 E-15$ \\
0.3 & $2.5 E-13$ & $9.74833 E-11$ & $9.74833 E-11$ & $9.9 E-16$ & $5.39165 E-14$ & $5.39165 E-14$ \\
\hline
\end{tabular}

Table 13 Numerical results for Example 5.2 with $\eta=0.06$

\begin{tabular}{llllll}
\hline $\boldsymbol{\tau}$ & ES & AS & AE & RE & $\begin{array}{l}\text { Time } \\
\text { CPU (s) }\end{array}$ \\
\hline 0.02 & 0.07984560896434381352 & 0.079845608964343853426 & $3.99 \times 10^{-17}$ & $4.99 \times 10^{-16}$ & 0.889 \\
0.04 & 0.15962763841261813303 & 0.15962763841261815794 & $2.49 \times 10^{-17}$ & $1.56 \times 10^{-16}$ & 1.232 \\
0.06 & 0.23928281206851416623 & 0.23928281206851423765 & $7.14 \times 10^{-17}$ & $2.98 \times 10^{-16}$ & 0.920 \\
0.08 & 0.31874845652859878735 & 0.31874845652859888367 & $9.63 \times 10^{-17}$ & $3.02 \times 10^{-16}$ & 0.904 \\
0.1 & 0.39796279376194770105 & 0.39796279376194771082 & $9.77 \times 10^{-18}$ & $2.45 \times 10^{-17}$ & 0.858 \\
\hline
\end{tabular}


Table 14 Numerical results for Example 5.2 with $\eta=0.06$

\begin{tabular}{llllll}
\hline $\boldsymbol{\tau}$ & ES & AS & AE & RE & $\begin{array}{l}\text { Time } \\
\text { CPU (s) }\end{array}$ \\
\hline 0.02 & 0.079734118548588251664 & 0.079734118548588199339 & $5.23 \times 10^{-17}$ & $6.56 \times 10^{-16}$ & 0.811 \\
0.04 & 0.15940492340173257962 & 0.15940492340173286314 & $2.83 \times 10^{-16}$ & $1.77 \times 10^{-15}$ & 0.796 \\
0.06 & 0.23894940199533129661 & 0.23894940199533120491 & $9.17 \times 10^{-17}$ & $3.83 \times 10^{-16}$ & 0.889 \\
0.08 & 0.31830514045634341908 & 0.31830514045634405770 & $6.38 \times 10^{-16}$ & $2.0 \times 10^{-15}$ & 0.982 \\
0.1 & 0.39741061409807554739 & 0.39741061409807569743 & $1.5 \times 10^{-16}$ & $3.77 \times 10^{-16}$ & 0.811 \\
\hline
\end{tabular}

Table 15 Numerical results for Example 5.2 with $\eta=0.1$

\begin{tabular}{llllll}
\hline $\boldsymbol{\tau}$ & ES & AS & AE & RE & $\begin{array}{l}\text { Time } \\
\text { CPU (s) }\end{array}$ \\
\hline 0.02 & 0.079591154289758679228 & 0.079591154289759560107 & $8.8 \times 10^{-16}$ & $1.1 \times 10^{-14}$ & 0.874 \\
0.04 & 0.15911933466140392832 & 0.15911933466140305331 & $8.75 \times 10^{-16}$ & $5.49 \times 10^{-15}$ & 0.874 \\
0.06 & 0.23852186564169922344 & 0.23852186564169936963 & $1.46 \times 10^{-16}$ & $6.12 \times 10^{-16}$ & 0.920 \\
0.08 & 0.31773666512002042948 & 0.31773666512002071579 & $2.86 \times 10^{-16}$ & $9.01 \times 10^{-16}$ & 1.295 \\
0.1 & 0.396702532289366215 & 0.39670253228936612 & $9.43 \times 10^{-17}$ & $2.37 \times 10^{-16}$ & 0.874 \\
\hline
\end{tabular}

Table 16 Comparison of absolute errors for Example 5.2

\begin{tabular}{lllllll}
\hline $\boldsymbol{\tau}$ & $\mathbf{A E}$ & AE & AE & AE & AE & AE \\
& RKM & MDM & RKM & MDM & RKM & MDM \\
& $\boldsymbol{\eta}=\mathbf{0 . 0 6}$ & $\boldsymbol{\eta}=\mathbf{0 . 0 6}[\mathbf{3 0}]$ & $\boldsymbol{\eta}=\mathbf{0 . 0 8}$ & $\boldsymbol{\eta}=\mathbf{0 . 0 8}[\mathbf{3 0}]$ & $\boldsymbol{\eta}=\mathbf{0 . 1}$ & $\boldsymbol{\eta}=\mathbf{0 . 1}[\mathbf{3 0}]$ \\
\hline 0.02 & $3.99 E-17$ & $2.22045 E-16$ & $5.23 E-17$ & $4.49640 E-15$ & $8.8 E-16$ & $4.47420 E-14$ \\
0.04 & $2.49 E-17$ & $2.22045 E-16$ & $2.83 E-16$ & $4.44089 E-15$ & $8.75 E-16$ & $4.44644 E-14$ \\
0.06 & $7.14 E-17$ & $1.94289 E-16$ & $9.17 E-17$ & $4.38538 E-15$ & $1.46 E-16$ & $4.41314 E-14$ \\
0.08 & $9.63 E-17$ & $1.94289 E-16$ & $6.38 E-16$ & $4.38538 E-15$ & $2.86 E-16$ & $4.36318 E-14$ \\
0.1 & $9.77 E-18$ & $1.94289 E-16$ & $1.5 E-16$ & $4.32987 E-15$ & $9.43 E-17$ & $4.29656 E-14$ \\
\hline
\end{tabular}

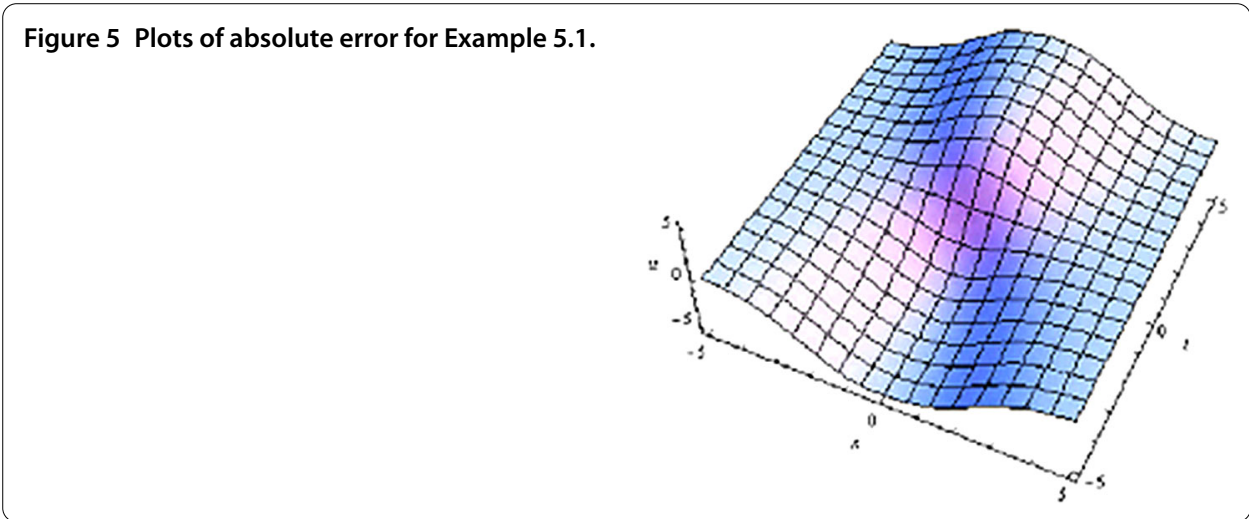

Example 5.2 We solve the SG equation (1) in the region $\Omega$ with the following initial conditions:

$$
u(\eta, 0)=0, \quad \frac{\partial u}{\partial \tau}(\eta, 0)=4 \sec h(\eta) .
$$

The exact solution is [27]

$$
u(\eta, \tau)=4 \arctan (\operatorname{sech}(\eta) \tau)
$$

After homogenizing the initial conditions by RKM, we get the results presented in Tables 4-16 and Figures 5-8. 


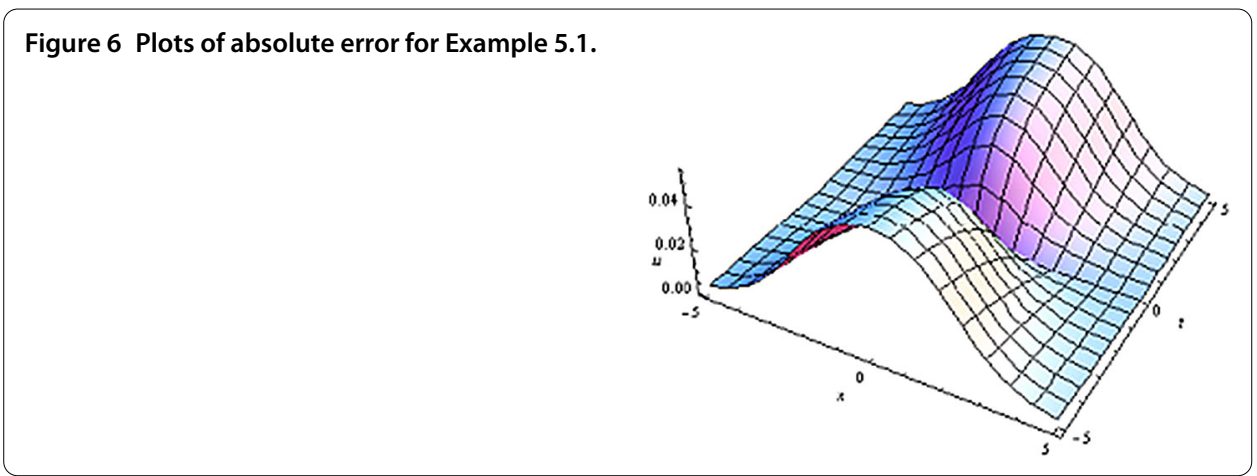

Figure 7 Plots of absolute error for Example 5.1.
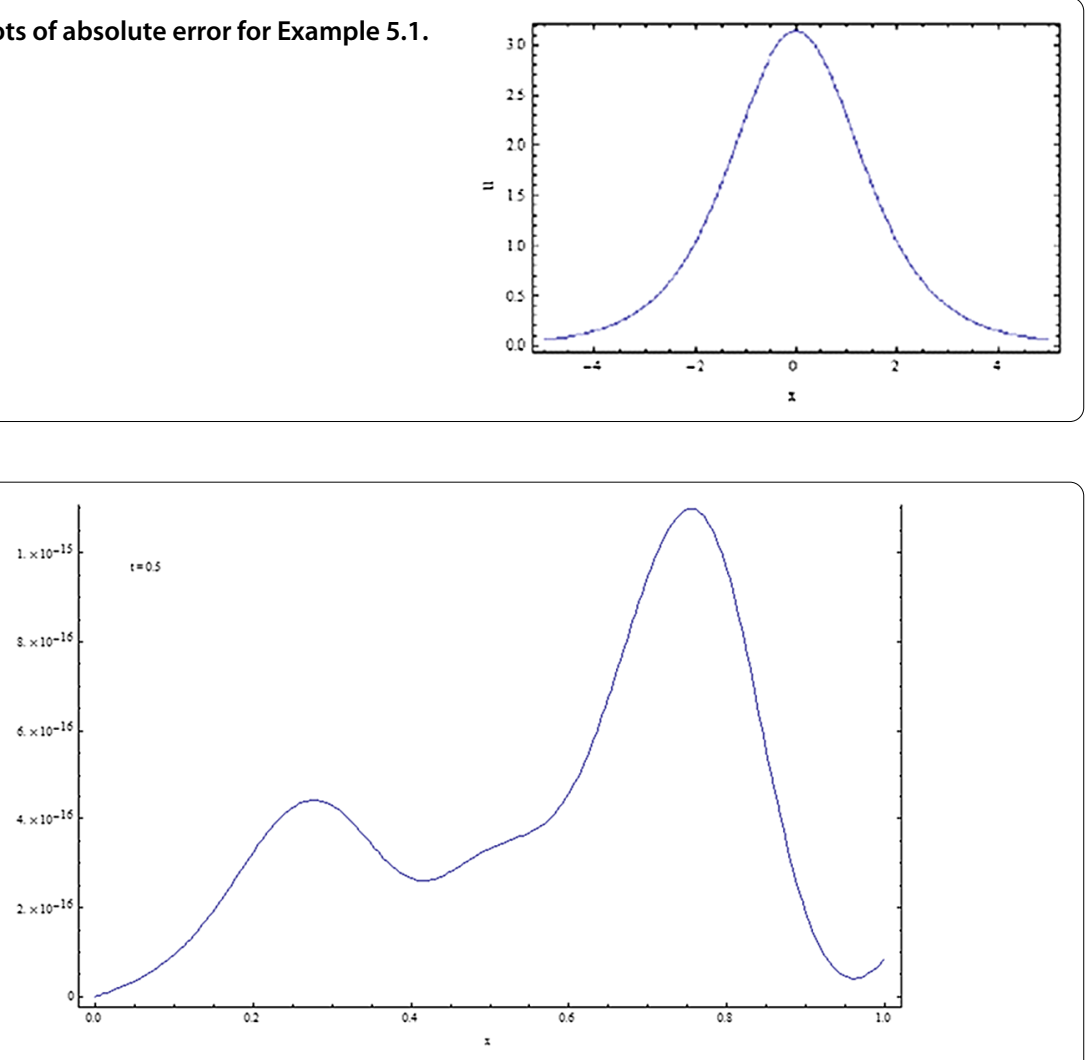

Figure 8 Plots of absolute error for Example 5.1.

Remark In Tables 1-16, we abbreviate the exact solution and the approximate solution by AS and ES, respectively. AE stands for the absolute error, that is, the absolute value of the difference of the exact and approximate solutions, whereas RE indicates the relative error, that is the absolute error divided by the absolute value of the exact solution.

\section{Conclusion}

Linear and nonlinear SG equations were investigated by RKM in this work. Homogenizing the initial and boundary conditions is very crucial for this method. We gave a general transformation to homogenize the conditions. This transformation will be very useful for researches who study RKM. We obtained very accurate numerical results and showed them by tables and figures. The computational results confirmed the efficiency, reliability, 
and accuracy of our method, which is easily applicable. RKM produced a rapidly convergent series with easily computable components using symbolic computation software. The results obtained by RKM are very effective and convenient with less computational work and time.

\section{Competing interests}

The authors declare that they have no competing interests.

\section{Authors' contributions}

All authors have read and approved the final manuscript.

\section{Author details}

'Department of Mathematics, Art and Science Faculty, Siirt University, Siirt, 56100, Turkey. ²Department of Mathematics, Science Faculty, Fırat University, Elazığ, 23119, Turkey. ${ }^{3}$ Department of Mathematics and Institute for Mathematical Research, University Putra Malaysia, Selangor, 43400, Malaysia. ${ }^{4}$ Department of Mathematics and Computer Sciences, Faculty of Arts and Sciences, Çankaya University, Ankara, 0630, Turkey. ${ }^{5}$ Department of Mathematics, Institute of Space Sciences, Magurele, Bucharest, Romania.

\section{Acknowledgements}

This paper is a part of the PhD thesis of the first author. The authors would like to thank to the referees for their very useful comments and remarks.

\section{Received: 15 September 2015 Accepted: 21 December 2015 Published online: 08 January 2016}

\section{References}

1. Polyanin, AD, Valentin, FZ: Handbook of Nonlinear Partial Differential Equations. Chapman \& Hall/CRC, Boca Raton (2004) xx+814 pp. ISBN:1-58488-355-3

2. Ramamurti, R: Solitons and instantons. In: An Introduction to Solitons and Instantons in Quantum Field Theory. North-Holland, Amsterdam (1982) viii+409 pp. ISBN:0-444-86229-3

3. Akbar, M, Dehghan, M: High-order solution of one-dimensional sine-Gordon equation using compact finite difference and DIRKN methods. Math. Comput. Model. 51(5-6), 537-549 (2010)

4. Scott, A: Nonlinear Science. Emergence and Dynamics of Coherent Structures, 2nd edn. Oxford Texts in Applied and Engineering Mathematics, vol. 8. Oxford University Press, Oxford (2003) xxiv+480 pp. ISBN:0-19-852852-3

5. Dauxois, T, Peyrard, M: Physics of Solitons. Cambridge University Press, Cambridge (2006)

6. Dehghan, M, Shokri, A: A numerical method for one-dimensional nonlinear sine-Gordon equation using collocation and radial basis functions. Numer. Methods Partial Differ. Equ. 24(2), 687-698 (2008)

7. Dehghan, M, Davoud, M: The boundary integral equation approach for numerical solution of the one-dimensional sine-Gordon equation. Numer. Methods Partial Differ. Equ. 24(6), 1405-1415 (2008)

8. Bratsos, AG: A third order numerical scheme for the two-dimensional sine-Gordon equation. Math. Comput. Simul. 76(4), 271-282 (2007)

9. Bratsos, AG: A numerical method for the one-dimensional sine-Gordon equation. Numer. Methods Partial Differ. Equ. 24(3), 833-844 (2008)

10. Dehghan, M, Shokri, A: A numerical method for solution of the two-dimensional sine-Gordon equation using the radial basis functions. Math. Comput. Simul. 79(3), 700-715 (2008)

11. Lakestani, M, Dehghan, M: Collocation and finite difference-collocation methods for the solution of nonlinear Klein-Gordon equation. Comput. Phys. Commun. 181(8), 1392-1401 (2010)

12. Dehghan, M, Farhad, Fl: The spectral collocation method with three different bases for solving a nonlinear partial differential equation arising in modeling of nonlinear waves. Math. Comput. Model. 53(9-10), 1865-1877 (2011)

13. Ahmed, H, Ahmed, A: Chebyshev collocation spectral method for solving the RLW equation. Int. J. Nonlinear Sci. 7(2), 131-142 (2009)

14. Ma, ML, Wu, ZM: A numerical method for one-dimensional nonlinear sine-Gordon equation using multiquadric quasi-interpolation. Chin. Phys. B 18, 3099-3103 (2009)

15. Aronszajn, N: Theory of reproducing kernels. Trans. Am. Math. Soc. 68, 337-404 (1950)

16. Cui, M, Lin, Y: Nonlinear Numerical Analysis in the Reproducing Kernel Space. Nova Science Publishers, New York (2009) xiv+226 pp. ISBN:978-1-60456-468-6; 1-60456-468-7

17. Geng, F, Cui, M, Zhang, B: Method for solving nonlinear initial value problems by combining homotopy perturbation and reproducing kernel Hilbert spaces methods. Nonlinear Anal., Real World Appl. 11, 637-644 (2010)

18. Mohammadi, M, Mokhtari, R: Solving the generalized regularized long wave equation on the basis of a reproducing kernel space. J. Comput. Appl. Math. 235, 4003-4014 (2011)

19. Jiang, W, Lin, Y: Representation of exact solution for the time-fractional telegraph equation in the reproducing kernel space. Commun. Nonlinear Sci. Numer. Simul. 16, 3639-3645 (2011)

20. Wang, Y, Su, L, Cao, X, Li, X: Using reproducing kernel for solving a class of singularly perturbed problems. Comput. Math. Appl. 61, 421-430 (2011)

21. $\mathrm{Wu}, \mathrm{B}, \mathrm{Xiu}, \mathrm{YL}$ : A new algorithm for a class of linear nonlocal boundary value problems based on the reproducing kernel method. Appl. Math. Lett. 24, 156-159(2011)

22. Yao, H, Lin, Y: New algorithm for solving a nonlinear hyperbolic telegraph equation with an integral condition. Int J. Numer. Methods Biomed. Eng. 27, 1558-1568 (2011)

23. Inc, M, Akgül, A: The reproducing kernel Hilbert space method for solving Troesch's problem. J. Assoc. Arab Univ. Basic Appl. Sci. 14, 19-27 (2013) 
24. Inc, M, Akgül, A, Geng, F: Reproducing kernel Hilbert space method for solving Bratu's problem. Bull. Malays. Math. Soc. 38(1), 271-287 (2015)

25. Inc, M, Akgül, A, Kilicman, A: Explicit solution of telegraph equation based on reproducing kernel method. J. Funct. Spaces Appl. 2012, Article ID 984682 (2012)

26. Zhang, S, Lei, L, LuHong, D: Reproducing kernel functions represented by form of polynomials. In: Proceedings of the Second Symposium International Computer Science and Computational Technology (ISCSCT '09), Huangshan, P.R. China, 26-28 December, pp. 353-358 (2009)

27. Sari, M, Gürhan, G: A sixth-order compact finite difference method for the one-dimensional sine-Gordon equation. Int. J. Numer. Methods Biomed. Eng. 27(7), 1126-1138 (2011)

28. Jiang, ZW, Wang, RH: Numerical solution of one dimensional sine-Gordon equation using high accuracy multiquadratic quasi-interpolation. Appl. Math. Comput. 218(15), 7711-7716 (2012)

29. Lu, J: An analytic approach to the sine-Gordon equation using the modified homotopy perturbation method. Comput. Math. Appl. 58, 2313-2319 (2009)

30. Kaya, D: A numerical solution of the sine-Gordon equation using the modified decomposition method. Appl. Math. Comput. 143, 309-317 (2003)

Submit your manuscript to a SpringerOpen ${ }^{\odot}$ journal and benefit from:

- Convenient online submission

Rigorous peer review

- Immediate publication on acceptance

- Open access: articles freely available online

- High visibility within the field

- Retaining the copyright to your article 